\title{
A PATIENT WITH DERMATOMYOSITIS SINE DERMATITIS AND NEUROMYOSITIS: COINCIDENCE OR ASSOCIATION?
}

Ana Woo Sook Kim¹, Isabela Bruna Pires Borges ${ }^{1}$, Rafael Giovani Misse ${ }^{1}$, Alexandre Moura dos Santos ${ }^{1}$, Fernando Henrique Carlos de Souza ${ }^{1}$, Samuel Katsuyuki Shinjo ${ }^{1, \star}$

1. Faculdade de Medicina FMUSP, Universidade de São Paulo, São Paulo (SP), Brazil.

*Corresponding author: samuel.shinjo@usp.br

\section{BACKGROUND}

Patients with Takayasu's arteritis (TAK) have several symptoms that can hamper their quality of life. However, little is known about the prevalence of chronic fatigue syndrome (CFS) and its contribution to reducing the quality of life and the daily activity levels (ADLs) in these patients.

\section{METHODS}

This cross-sectional study included 53 TAK patients (ACR, 1990) were who age and gender-matched to 100 healthy individuals, from 2020 to 2021. Disease activity was assessed by ITAS2010. The ADLs was assessed by HAQ; physical activity was assessed by IPAQ-SF, whereas CFS was assessed using the fatigue severity scale (FSS), modified fatigue impact scale (MFIS), and analogue visual scale for fatigue.

\section{RESULTS}

Patients with TAK and control individuals had comparable current age (43.0 [38.5-51.5] vs. 49.0 [37.6-55.8] years), body mass index, and gender distribution. The median disease duration was 13.0 (6.5-20.0) years, and 11 (20.8\%) patients had disease activity. In addition, patients had a significant higher prevalence of CFS, but lower ADLs, physical activity level, and the physical and psychosocial domains of MFIS, compared to healthy individuals (Figure 1). Patients with TAK were approximately 2.5 times more likely to have CFS in relation to control individuals (FFS: $\mathrm{OR}=2.373 ; 95 \% \mathrm{Cl}=1.170-4.829$; and $\mathrm{MFIS}=2.61795 \% \mathrm{Cl}=1.283-5.249$ ). Moreover, the CFS correlated to worsening HAQ, daily prednisone dose, and disease activity (Figure 2) In addition, CFS correlated with the number of immunosuppressive drugs used (RPB $=0.449 ; p=0.003$ ).

(1)

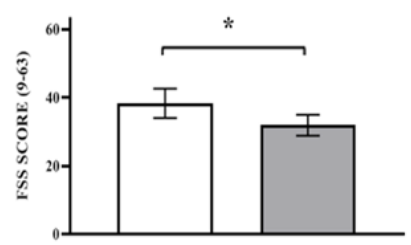

(2)

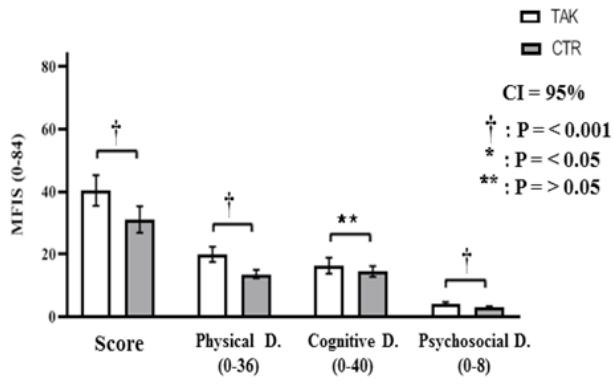

(3)

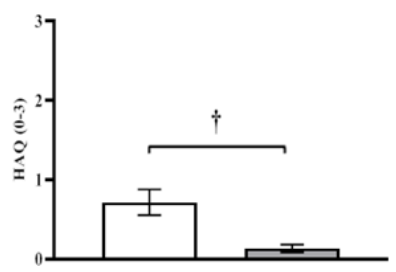

(4)

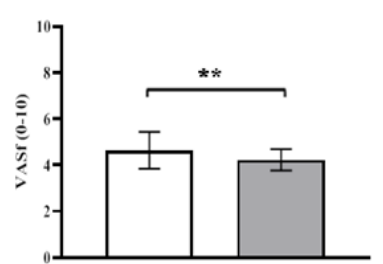

(5)

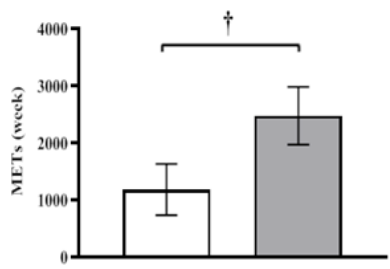

Figure 1. Fatigue, activities of daily living, physical activity of patients with Takayasu's arteritis and the healthy individuals.

CTR: Healthy individuals; FSS: Fatigue severity scale; HAQ: Health assessment questionnaire; METs: Metabolic equivalent for week; MFIS: Modified fatigue impact scale; TAK: Takayasu's arteritis; VASf: Visual analogic scale for fatigue. 


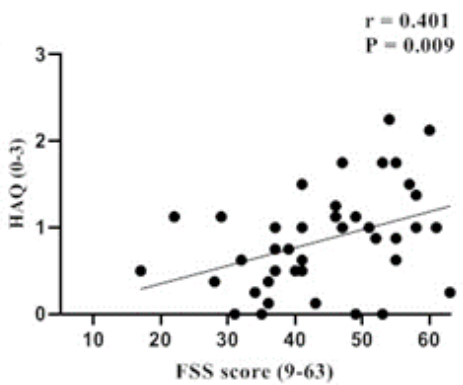

(4)

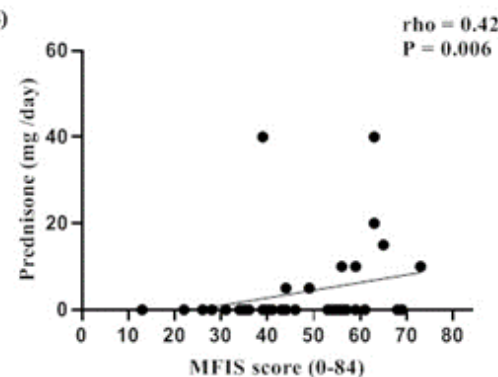

(2)

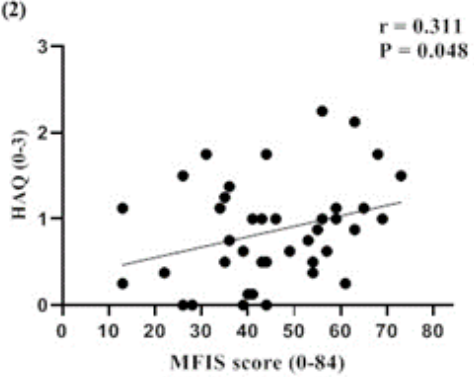

(5)

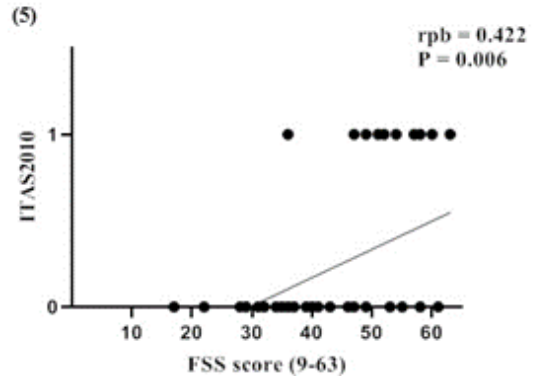

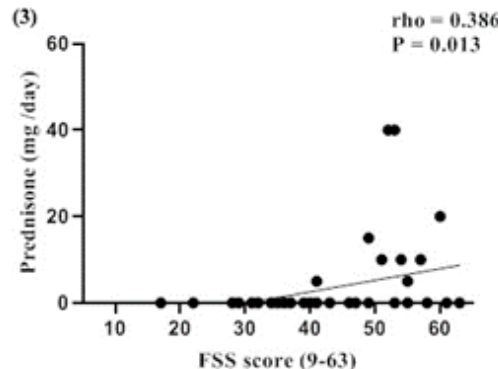

(6)

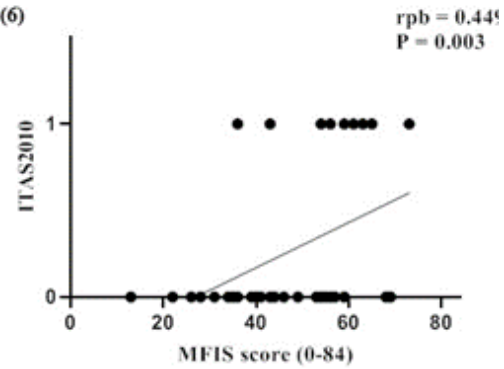

Figure 2. Correlations between fatigue, prednisone dose use, disease activity, and activities of daily living.

\section{CONCLUSION}

The TAK patients have a high prevalence of CFS with possible interference in the ADLs. Therefore, the CFS-focused treatments, such as pharmacological/nonpharmacological treatments, are also should be considered in clinical practice.

\section{FUNDING}

Support by FAPESP: Grant No. 2018/0873533 to AMS;2019/12155-5 to RGM;2019/11776-6 to SKS; CNPq Grant No. 303379/2018-9 to SKS.

\section{KEYWORDS}

Takayasu's arteritis, Chronic fatigue, Patient outcomes, Quality of life. 